Check for updates

Cite this: Phys. Chem. Chem. Phys. 2021, 23, 13680

Received 3rd May 2021,

Accepted 4th June 2021

DOI: $10.1039 / \mathrm{d} 1 \mathrm{cp} 01960 \mathrm{k}$

rsc.li/pccp

\title{
Vacancy diffusion in palladium hydrides $\dagger$
}

\author{
Jonathan M. Polfus, (D) *ab Thijs Peters, (D) ${ }^{\text {a }}$ Rune Bredesen ${ }^{\mathrm{a}}$ and \\ Ole Martin Løvvik (D) *a
}

\begin{abstract}
The self-diffusion coefficients of palladium in $\mathrm{PdH}_{x}(x=0,0.25,0.5,0.75,1)$ were studied using density functional theory to obtain the required thermodynamic and kinetic parameters. The enthalpy of migration decreased from $0.95 \mathrm{eV}$ for $\mathrm{Pd}$ to $0.78 \mathrm{eV}$ for $\mathrm{PdH}$. The enthalpy of vacancy formation exhibited a substantial decrease from about $1.1 \mathrm{eV}$ in $\mathrm{Pd}$ to $0.4 \mathrm{eV}$ in $\mathrm{PdH}$, which was ascribed to successive filling of antibonding states weakening the $\mathrm{Pd}-\mathrm{Pd}$ bonds. Concurrently, the Arrhenius pre-exponential was significantly reduced from $4.75 \times 10^{-3} \mathrm{~cm}^{2} \mathrm{~s}^{-1}$ for $P d$ to $5.67 \times 10^{-9} \mathrm{~cm}^{2} \mathrm{~s}^{-1}$ for $\mathrm{PdH}$ due to softening of the vibrational modes that determine the entropy of vacancy formation and initial/transition state frequencies. A linear correlation between the logarithm of the pre-exponential and the activation energy was interpreted as enthalpy-entropy compensation (Meyer-Neldel rule). The Pd self-diffusion coefficients in the hydrides were within 1 order of magnitude of that in pure palladium above $200{ }^{\circ} \mathrm{C}$ for hydrogen pressures up to at least $10^{7} \mathrm{~Pa}$.
\end{abstract}

\section{Introduction}

Palladium exhibits fast dissociation kinetics of molecular hydrogen at its surfaces and high bulk diffusivity of atomic hydrogen. Combined, these properties constitute the basis for the functional properties of Pd-based materials in several applications. Most notably, Pd-based alloys have been employed as $\mathrm{H}_{2}$ selective membranes for gas separation and membrane reactors, ${ }^{1}$ as well as fiber-optic $\mathrm{H}_{2}$ sensors. ${ }^{2}$ Recently, palladium has also been applied as interfacial electrode layer in electrochemical energy conversion devices based on proton-conducting ceramics, ${ }^{3}$ and as hydrogen reservoir and gate electrode in a protonic solid-state electrochemical synapse. ${ }^{4}$

Hydrogen absorbs into the octahedral interstitial sites of the fcc structure of palladium and causes expansion of the lattice as well as changes to the electron and phonon band structures. ${ }^{5}$ However, exposure to hydrogen can as well result in a range of structural and microstructural deterioration processes, including embrittlement, cracking and void formation, ${ }^{6-8}$ which may be ascribed to several aspects. The $\mathrm{Pd}-\mathrm{H}$ phase diagram exhibits a miscibility gap below $290{ }^{\circ} \mathrm{C}$ between a diluted $\mathrm{PdH}_{x}$ phase $(\alpha)$ and a concentrated phase $(\beta, 1 \geq x \gtrsim 0.6$ at room temperature). Exposure to hydrogen can thus result in phase separation and

\footnotetext{
${ }^{a}$ SINTEF Industry, Sustainable Energy Technology, PO Box 124 Blindern,

Oslo NO-0314, Norway. E-mail: olemartin.lovvik@sintef.no

${ }^{b}$ Department of Chemistry, Centre for Materials Science and Nanotechnology,

University of Oslo, PO Box 1033 Blindern, Oslo N-0315, Norway.

E-mail: jonathan.polfus@kjemi.uio.no

$\dagger$ Electronic supplementary information (ESI) available. See DOI: 10.1039/ d1cp01960k
}

hysteresis in the absorption/desorption isotherms. ${ }^{9}$ The presence of hydrogen further leads to increased concentrations of metal vacancies due to favorable interactions with hydrogen interstitials through formation of vacancy-hydrogen clusters. ${ }^{10-12}$ Despite their abundance, monovacancies do not spontaneously coalesce into larger voids due to the significant loss of configurational entropy that would be associated with clustering. ${ }^{12}$ Void formation therefore appears to be caused by other factors such as decohesion related to so-called hydrogen-enhanced local plasticity. ${ }^{13}$

Self-diffusion of palladium is central to most of the aforementioned degradation processes and can govern the degradation rate and lifetime of the material or device ${ }^{8,14}$ Dissolution of hydrogen has been reported to result in hydrogen-induced lattice migration and diffusion creep in palladium. ${ }^{15-17}$ Lacking still are self-diffusion coefficients of palladium in palladium hydrides, and a deeper understanding of their dependence on temperature and composition. Metal diffusion proceeds according to the vacancy mechanism in palladium, and it is reasonable to expect the same mechanism to dominate in palladium hydrides considering their common fcc arrangement of Pd atoms. The diffusivity follows an Arrhenius behavior and can be expressed as

$$
D=D_{0} \exp \left(\frac{E_{\mathrm{a}}}{k_{\mathrm{B}} T}\right)
$$

where the activation energy $E_{\text {a }}$ comprises the enthalpies of formation and migration of monovacancies, $\Delta H^{\mathrm{f}}$ and $\Delta H^{\mathrm{m}}$, respectively, $k_{\mathrm{B}}$ is the Boltzmann constant, and $T$ is temperature. 


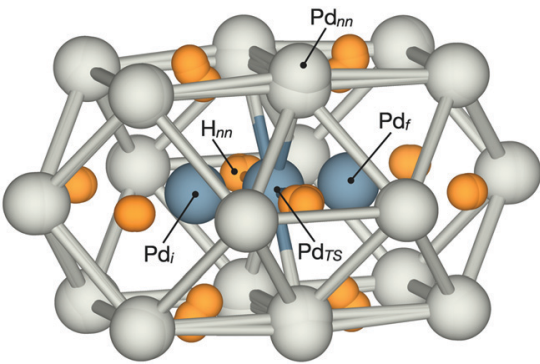

Fig. 1 Local structure of a migrating $\mathrm{Pd}$ atom in $\mathrm{PdH}$ at the initial state $\left(\mathrm{Pd}_{\mathrm{i}}\right)$, transition state $\left(\mathrm{Pd}_{\mathrm{TS}}\right)$ and final state $\left(\mathrm{Pd}_{\mathrm{f}}\right) . \mathrm{Pd}_{\mathrm{Ts}}$ is coordinated to four $\mathrm{Pd}$ atoms in a square-planar configuration $\left(\mathrm{Pd}_{\mathrm{nn}}\right)$ and two hydrogen atoms $\left(\mathrm{H}_{n n}\right)$. The local relaxation during migration is shown by superposition of the atomic positions.

The pre-exponential factor $D_{0}$ can be expressed as

$$
D_{0}=f a^{2} v^{*} \exp \left(\frac{\Delta S_{\mathrm{vib}}^{\mathrm{f}}}{k_{\mathrm{B}}}\right)
$$

where the correlation factor $f$ is 0.7815 for vacancy diffusion in fcc lattices, ${ }^{18} a$ is the lattice constant and $\Delta S_{\mathrm{vib}}^{\mathrm{f}}$ is the entropy of formation of monovacancies. As described by transition state theory, ${ }^{19}$ the effective frequency $v^{*}$ is given by the ratio of the products of the vibrational frequencies at the initial $\left(v_{i}\right)$ and transition state $\left(v_{i}^{\prime}\right)$, according to

$$
v^{*}=\prod_{i=1}^{N} v_{i} / \prod_{i=1}^{N-1} v_{i}^{\prime}
$$

where the imaginary frequency along the migration direction at the transition state is notably not included.

Self-diffusion coefficients of palladium in palladium hydrides are not readily attainable by conventional experimental methods due to the limited stability of the materials at the temperatures required for appreciable diffusion to occur. However, density functional theory (DFT) calculations can be used to directly obtain the required thermodynamic and kinetic parameters according to the above set of equations. ${ }^{20}$ Accordingly, the present work provides self-diffusion coefficients in palladium hydrides including the underlying contributions from both formation and migration of vacancies, as illustrated in Fig. 1. Furthermore, the study provides detailed insight into the relationship between the self-diffusion coefficients and the physical properties of the materials, notably lattice dynamics and electronic structure.

\section{Methods}

DFT calculations were performed using the projector augmented wave method implemented in the Vienna ab initio simulation package (VASP) and the generalized gradient approximation due to Perdew, Burke and Ernzerhof (GGA-PBE). ${ }^{21,22}$ The calculations were carried out with a plane wave cut-off energy of $500 \mathrm{eV}$ and an electronic convergence criterion of $10^{-6} \mathrm{eV}$. In addition to Pd and $\mathrm{PdH}, \mathrm{PdH}_{x}(x=0.25,0.5,0.75)$ were constructed as special quasirandom structures,${ }^{23}$ in line with the disordered nature of interstitial hydrogen at ambient and elevated temperatures. A $\Gamma$-centered $2 \times 2 \times 2$ Monkhorst-Pack $k$-point grid was used for the $4 \times 4 \times 4$ supercells containing 256-512 atoms. Migration barriers were calculated using the climbing image nudged elastic band (CI-NEB) method. ${ }^{24}$ The atomic positions were relaxed until the residual forces were within $0.02 \mathrm{eV} \AA^{-1}\left(0.03 \mathrm{eV} \AA^{-1}\right.$ for selected CI-NEB calculations). Vibrational frequencies were obtained with the finite displacement method and harmonic approximation.

The vacancy formation enthalpies $\Delta H^{\mathrm{f}}$ were determined from vacancy formation energies $\left(\Delta E^{f}\right)$ and zero-point energy (ZPE) contributions from $\mathrm{H}$ and Pd according to

$$
\Delta H^{\mathrm{f}}=\Delta E^{\mathrm{f}}+n \Delta \mathrm{ZPE}_{\mathrm{H}}+l \Delta \mathrm{ZPE}_{\mathrm{Pd}}
$$

where $n$ and $l$ are the number of hydrogen and palladium atoms adjacent to the vacancy, respectively. The ZPE contribution from changes in the vibrational frequencies of the Pd atoms $l \Delta \mathrm{ZPE}_{\mathrm{Pd}}$ was determined to be insignificant and not considered further, e.g., $-6 \mathrm{meV}$ for a the 12 coordinated monovacancy in Pd. Vibrational entropies were calculated from the vibrational frequencies of the atomic species according to

$$
S_{\text {vib }}=k_{\mathrm{B}} \sum_{j}\left(\frac{\beta_{j}}{\exp \left(\beta_{j}\right)-1}-\ln \left(1-\exp \left(-\beta_{j}\right)\right)\right)
$$

where $\beta_{j}=h v_{j} / k T$ and $v_{j}$ are the vibrational frequencies. The entropy of formation of the vacancies was calculated according to

$$
\Delta S_{\text {vib }}^{\mathrm{f}}=l \Delta S_{\mathrm{v}}^{\mathrm{f}}+n \Delta S_{\mathrm{H}}^{\mathrm{f}}
$$

where $\Delta S_{\mathrm{v}}^{\mathrm{f}}$ was calculated as the difference in vibrational entropy for a Pd atom adjacent to a vacancy and a bulk Pd atom. Additional computational details have been reported previously. ${ }^{12}$

\section{Results and discussion}

The optimized lattice parameters showed a linear expansion with hydrogen content according to Vegard's law, from $3.94 \AA$ for Pd to $4.13 \AA$ for PdH (ESI $\dagger$ Fig. S1). The enthalpy of vacancy formation exhibited a strong dependence on composition, decreasing from about $1.1 \mathrm{eV}$ in $\mathrm{Pd}$ to $0.4 \mathrm{eV}$ in $\mathrm{PdH}$ (Fig. 2(a)). While the zero-point energy contribution to $\Delta H^{\mathrm{f}}$ was essentially zero from the Pd atoms adjacent to the vacancy, there was a substantial contribution from hydrogen due to its low mass. Notably, the magnitude of $\triangle \mathrm{ZPE}$ increased as a function of hydrogen content, and the total contribution eventually amounted to half of the vacancy formation enthalpy for PdH with six hydrogen atoms adjacent to the vacancy. The vacancies are stabilized by clustering with interstitial hydrogen, but the considered vacancies in $\mathrm{PdH}_{0.25}$ were associated with an average of only one hydrogen atom. These vacancies may therefore be further stabilized by clustering with additional hydrogen atoms. Still, vacancy-hydrogen clustering is less important at higher temperatures where configurational entropy prevails. For instance, vacancy clusters with 1-2 

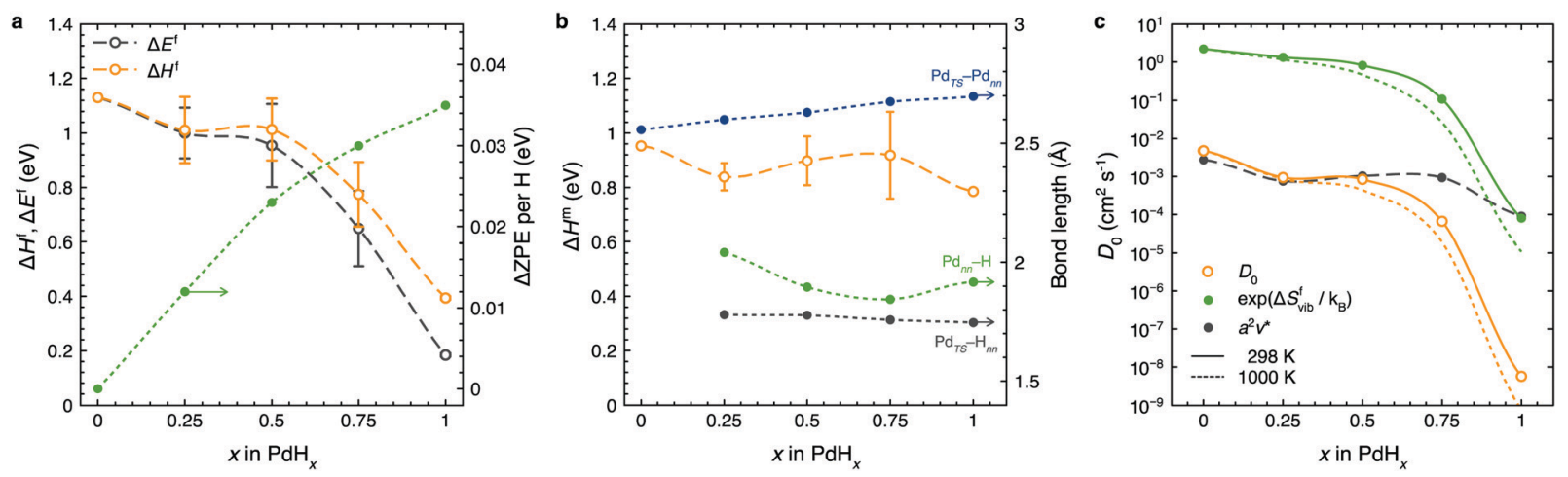

Fig. 2 Contributions to the self-diffusion coefficient as a function of $\mathrm{PdH}_{x}$ composition: (a) enthalpy of vacancy formation $\Delta H^{f}$ and contribution from zero-point energy $\Delta \mathrm{ZPE}$ per $\mathrm{H}$. (b) Enthalpy of migration $\Delta H^{\mathrm{m}}$ and average bond lengths to nearest $\mathrm{Pd}$ and $\mathrm{H}$ at the transition state, and minimum distance between $\mathrm{Pd}_{\mathrm{nn}}$ and adjacent hydrogen atoms. (c) Pre-exponential factor $D_{0}$ and the contribution from entropy of vacancy formation $\Delta S_{\text {vib }}^{f}$ and effective frequency $v^{*}$, with temperature-dependent variables shown for $298 \mathrm{~K}$ and $1000 \mathrm{~K}$. The error bars are standard deviations for five different vacancy sites in (a) and the corresponding four transitions states in (b) $(E S I \dagger)$. Lines are guides to the eye.

hydrogen atoms were found to predominate above approximately $160{ }^{\circ} \mathrm{C}$ in palladium. ${ }^{12}$

The origin of the large variation in enthalpy of vacancy formation for $\mathrm{PdH}_{0.75}$ and $\mathrm{PdH}$ (Fig. 2(a)) can be understood from the electronic structure of the materials. Fig. 3 shows the electronic density of states of the palladium hydrides and the corresponding Fermi levels. Notably, the Fermi level is shifted to higher energies from $\mathrm{PdH}_{0.75}$, resulting in a destabilization of the system by successive filling of antibonding states, specifically d band Pd $t_{2 \mathrm{~g}}-\mathrm{t}_{2 \mathrm{~g}}$ according to previous analysis. ${ }^{25}$ As these antibonding states are filled, it becomes increasingly easy to form vacancies.

The migration enthalpy $\Delta H^{\mathrm{m}}$ showed less variation with composition in comparison to the formation enthalpy, ranging from $0.95 \mathrm{eV}$ for Pd to $0.78 \mathrm{eV}$ for PdH (Fig. 2(b)). Moreover, the non-monotonic behavior as a function of hydrogen content indicates at least two competing effects. These can reasonably be ascribed to two structural features with opposite impact on $\Delta H^{\mathrm{m}}$ : while the distance between the migrating Pd atom and its nearest neighbors at the transition state $\left(\mathrm{Pd}_{\mathrm{TS}}-\mathrm{Pd}_{\mathrm{nn}}\right)$ increases with hydrogen content, the $\mathrm{Pd}_{\mathrm{nn}}$ atoms are simultaneously pushed closer to adjacent hydrogen atoms, especially for $\mathrm{PdH}_{0.5}$ and $\mathrm{PdH}_{0.75}$ (Fig. 2(b)). The latter aspect is subject to the presence of hydrogen at sites along the direction to which the $\mathrm{Pd}_{\mathrm{nn}}$ atoms are pushed, and therefore leads to a rather large variation in $\Delta H^{\mathrm{m}}$ for these compositions. In the case of $\mathrm{PdH}$, the expanded lattice appears to lead to less steric constraints of this kind.

The pre-exponential factor $D_{0}$ and its major contributions from the effective frequency $v^{*}$ and entropy of vacancy formation $\Delta S_{\text {vib }}^{\mathrm{f}}$ are shown in Fig. 2(c). The entropies of vacancy formation were determined from the vibrational frequencies of the nearest neighbor palladium and hydrogen atoms. On the other hand, the effective frequencies were determined from the vibrational frequencies of nearest neighbor Pd atoms (at the initial and transition state), while hydrogen was considered to be free to rearrange during the migration event. ${ }^{26}$ The entropy of vacancy formation exhibited a steep decrease for $\mathrm{PdH}$ which could be

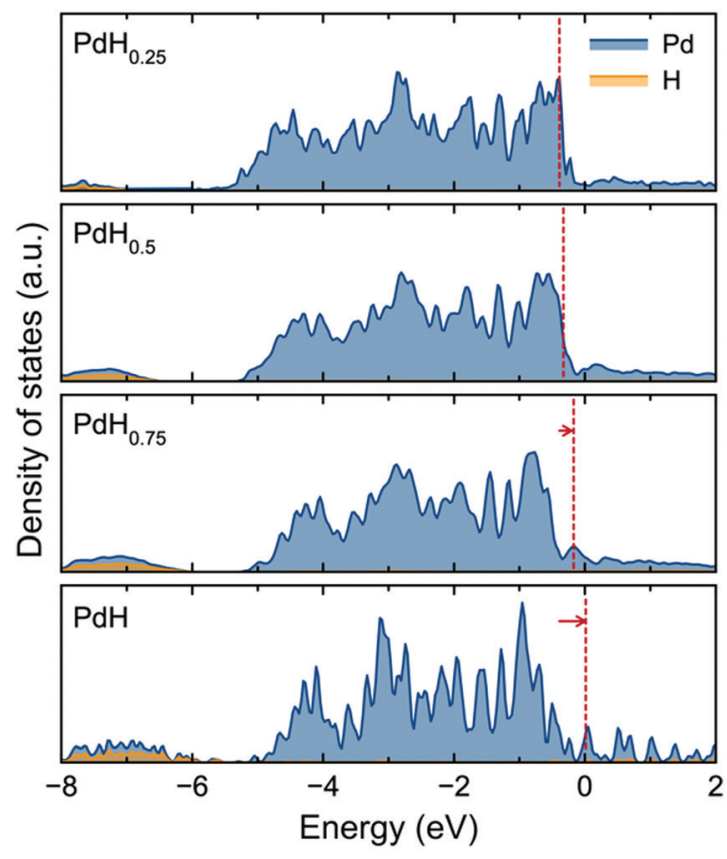

Fig. 3 Electronic partial density of states for $\mathrm{PdH}_{x}$ with the Fermi level indicated by red lines. The Fermi level in $\mathrm{PdH}$ was referenced to zero and core-level Pd states were used for alignment across compositions.

associated with changes in the vibrational entropies of both palladium and hydrogen atoms upon vacancy formation (ESI $\dagger$

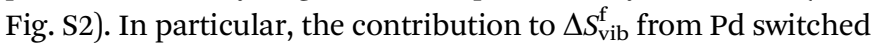
from positive to negative between $\mathrm{PdH}_{0.75}$ and $\mathrm{PdH}$, most likely due to the weakening of the bulk Pd-Pd bonds as the antibonding states were filled. The contribution to $\Delta S_{\text {vib }}^{\mathrm{f}}$ from the hydrogen atoms was negative for all compositions since the $\mathrm{Pd}-\mathrm{H}$ bonds were shortened and the vibrational frequencies of hydrogen increased adjacent to the vacancy (ESI $\dagger$ Table S3). The effective frequency showed a limited variation of 1.5 orders of magnitude as a function of hydrogen content. Notably, the effective frequency followed the same trend as $\Delta H^{\mathrm{m}}$, which may be understood from 


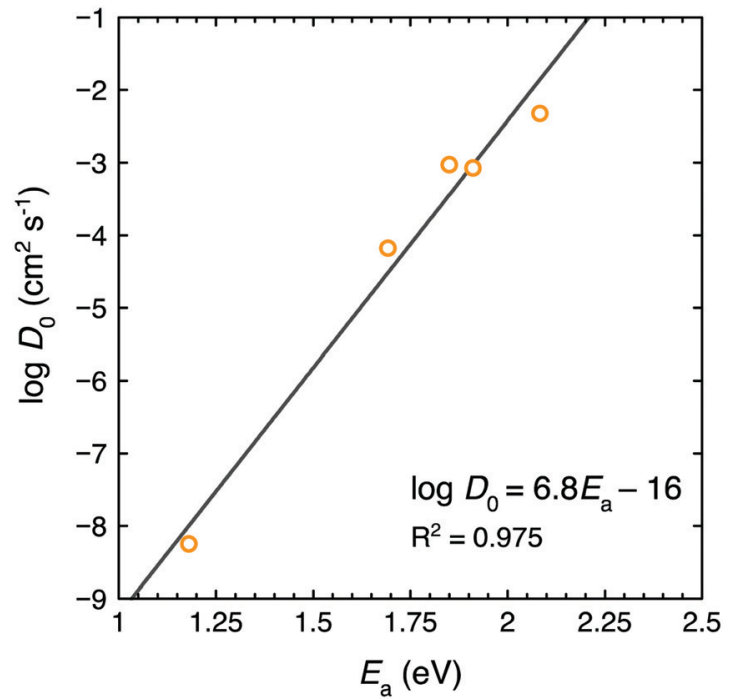

Fig. 4 Correlation between the pre-exponential factor $D_{0}(298.15 \mathrm{~K})$ and activation energy $\left(\Delta H_{f}+\Delta H_{m}\right)$ for $\mathrm{PdH}_{x}$ with linear fit.

the relationship between the bond lengths and dynamics of the palladium atoms at the transition state. Combined, these contributions resulted in a pre-exponential for $\mathrm{PdH}$ that is five orders of magnitude lower than for $\mathrm{Pd}$ and $\mathrm{PdH}_{x}$ up to $x=0.5$. The vacancy formation entropy showed relatively little variation with temperature, which translates to a minor non-linearity in the Arrhenius behavior of the diffusion coefficients.

Correlations between the pre-exponential factor $D_{0}$ and the enthalpic terms in the activation energy were considered based on the observation of so-called enthalpy-entropy compensation
(Meyer-Neldel rule) in a wide range of thermally activated processes. In particular, enthalpy-entropy compensation has been reported for self- and impurity diffusion in metals and on metal surfaces, ${ }^{27,28}$ hydrogen diffusion in minerals, ${ }^{29}$ and oxygen diffusion in perovskite oxides. ${ }^{30}$ As shown in Fig. 4, a linear correlation was obtained between $\log D_{0}$ and the activation energy. The correlation with $\log D_{0}$ was similar for $\Delta H_{\mathrm{f}}$ while it was not evident for $\Delta H_{\mathrm{m}}$. The compensation effect has been largely ascribed to softening of low-frequency modes at the transition state relative to the initial state based on diffusion studies in silicon and aluminum. ${ }^{31}$ In the present case, however, the change in $D_{0}$ was mainly determined by the change in the entropy of vacancy formation, which in turn had a significant contribution from the vibrational entropy of hydrogen (ESI $\dagger$ Fig. S2). The contribution to $D_{0}$ from the relative softening of the vibrational modes at the transition state, i.e., the change in effective frequency, was less pronounced (Fig. $2 \mathrm{c}$ and ESI $\dagger$ Table S3).

The diffusion coefficients are summarized for the given hydride compositions in the Arrhenius plots in Fig. 5(a). PdH exhibits a distinctly lower activation energy, but also a significantly lower pre-exponential factor than the other compositions, and the resulting diffusivity is higher than that of Pd below $400{ }^{\circ} \mathrm{C}$. The calculated diffusion coefficient of pure palladium is in reasonable agreement with experimental values, although both the pre-exponentials and the activation energies are somewhat different (Fig. 5(a), inset). These discrepancies may be associated with the anomalous non-linear elastic behavior of palladium with a transition at around $600{ }^{\circ} \mathrm{C},{ }^{32}$ or intrinsic surface errors in the vacancy calculations. ${ }^{33}$ The obtained enthalpies of formation and migration of vacancies in Pd are in good agreement with similar computational studies. ${ }^{34}$

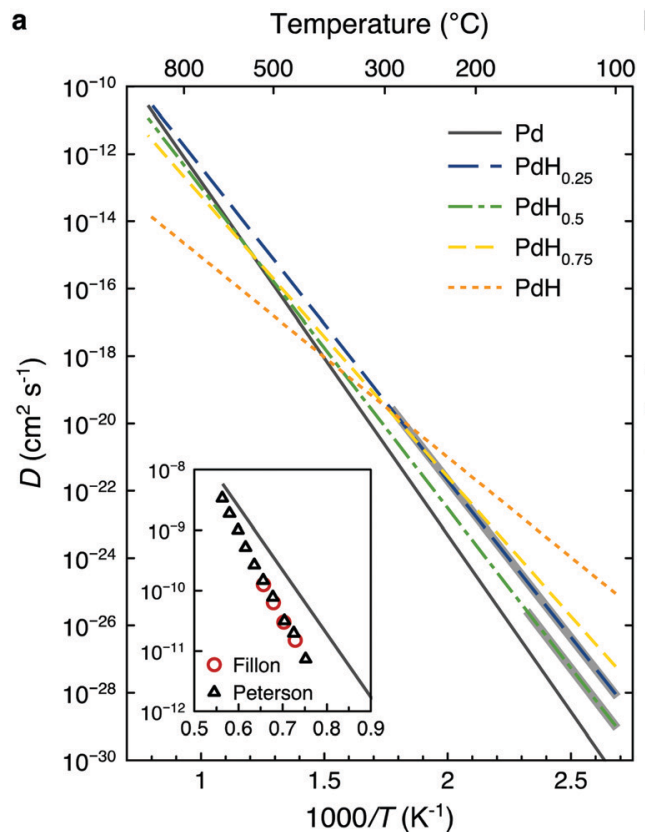

b
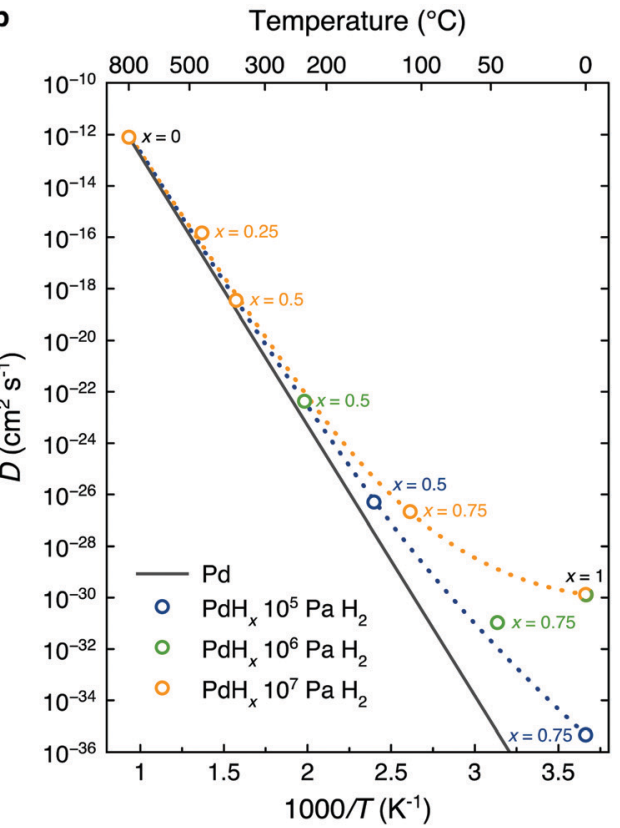

Fig. 5 Arrhenius plot of the diffusion coefficients for fixed $\mathrm{PdH}_{x}$ compositions (a) and for isobaric conditions (b). The inset in (a) shows a comparison between the calculated self-diffusion coefficient in pure Pd and experimental values from ${ }^{103} \mathrm{Pd}$ impurity diffusion in single- and polycrystalline Pd by Peterson ${ }^{36}$ and Fillon. ${ }^{37}$ The shaded regions for $\mathrm{PdH}_{0.25}$ and $\mathrm{PdH}_{0.5}$ in (a) are within the $\mathrm{Pd}-\mathrm{H}$ miscibility gap. The dotted lines in (b) are guides to the eye. 
In evaluating the diffusion coefficients in palladium hydrides, it may be most relevant to consider isobaric conditions. As shown in Fig. 5(b), the considered compositions correspond to a single temperature at the given $\mathrm{H}_{2}$ pressures, outside of the miscibility gap in the $\mathrm{Pd}-\mathrm{H}$ phase diagram. ${ }^{35}$ Here, the presence of hydrogen results in an increased diffusion coefficient at all temperatures as long as the hydrogen content is appreciable. The largest difference in diffusivity between Pd and its hydrides is obtained for $\mathrm{PdH}$ at the lowest temperatures. For instance, the diffusivity of $\mathrm{PdH}$ at $0{ }^{\circ} \mathrm{C}$ is the same as that of Pd at $109{ }^{\circ} \mathrm{C}$. Overall, it is reasonable to assert that the self-diffusion coefficients are within 1 order of magnitude of that of pure palladium above $200{ }^{\circ} \mathrm{C}$ for hydrogen pressures up to at least $10^{7} \mathrm{~Pa}$ (100 bar).

The softening of the vibrational modes of the palladium lattice upon hydride formation is well established, for instance in $\mathrm{PdH}_{0.66}{ }^{38}$ It may be noted that the calculations of the vibrational frequencies of hydrogen in the disordered structures, i.e., $\mathrm{PdH}_{0.25}, \mathrm{PdH}_{0.5}$ and $\mathrm{PdH}_{0.75}$, revealed anharmonicity that may be best described as asymmetry in the potential energy profile for hydrogen (ESI $\dagger$ Fig. S4). ${ }^{5}$ The disorder resulted in distortion of hydrogen to off-center positions, while hydrogen adjacent to metal vacancies relaxed to welldefined positions with more symmetric potential energy profiles.

Hydrogen embrittlement of palladium reflects the tendency for elastic breakdown and crack formation due to reduced ductility and enhanced localized plasticity in regions saturated with hydrogen. ${ }^{39}$ The formation of voids has therefore been ascribed to local enrichment of hydrogen that leads to decohesion due to the impaired mechanical properties. ${ }^{13}$ Accordingly, void formation at grain boundaries may be caused by segregation of hydrogen and vacancy-hydrogen clusters in these regions. ${ }^{8,12}$ Hydrogen can additionally affect the microstructural features of palladium such as increased dislocation densities, ${ }^{6}$ presumably influenced by chemical expansion and associated strain. While void formation may be further facilitated by microstrain, abundant monovacancies and clusters, ${ }^{11,12}$ the present work implies that enhanced vacancy diffusion is not a major factor in void formation.

The detrimental changes in mechanical properties upon hydride formation may be suppressed by addition of alloy elements. For instance, the expanded lattice and increased plasticity of palladium-silver alloys suppress the miscibility gap and internal stresses associated with the $\alpha / \beta$ phase transition. ${ }^{40,41}$ At the same time, alloying with $\mathrm{Ag}$ leads to filling of the Pd d band, ${ }^{42}$ and can thereby advance the filling of antibonding states upon hydrogen incorporation. Thus, alloying that leads to improved mechanical properties in relation to the $\alpha / \beta$ phase transition may also increase the susceptibility of the $\alpha$-phase towards decohesion in regions locally enriched in hydrogen. Further studies are required for quantitative assessments of the role of alloy elements and the degree of d-band filling on the mechanical properties of palladium hydrides.

\section{Conclusions}

The self-diffusion coefficients in the palladium hydride system exhibited a complex relationship between the hydrogen content and several concurrent changes in the physical properties of the materials, including bond lengths, unit cell volume, lattice dynamics, and electronic structure. In particular, the overall reduction in the enthalpies of formation and migration of vacancies with increasing hydrogen content was accompanied by a softening of the lattice vibrations that resulted in a significant reduction of the pre-exponential factor. Furthermore, the logarithm of the pre-exponential was determined to correlate linearly with the activation energy of diffusion, which was interpreted as enthalpy-entropy compensation. The resulting selfdiffusion coefficients were therefore lower than previous estimates based solely on changes in the vacancy concentration. ${ }^{43}$

\section{Conflicts of interest}

There are no conflicts to declare.

\section{Acknowledgements}

This work was supported by the Research Council of Norway, through the CLIMIT program (Contract No. 281824), and HYDROGEN Mem-Tech AS. Computational resources were provided by Uninett Sigma2 under the projects nn9259k and nn2615k.

\section{References}

1 N. A. Al-Mufachi, N. V. Rees and R. Steinberger-Wilkens, Hydrogen selective membranes: a review of palladiumbased dense metal membranes, Renewable Sustainable Energy Rev., 2015, 47, 540-551.

2 S. F. Silva, L. Coelho, O. Frazão, J. L. Santos and F. X. Malcata, A review of palladium-based fiber-optic sensors for molecular hydrogen detection, IEEE Sens. J., 2012, 12, 93-102.

3 J. S. Park, H. J. Choi, G. D. Han, J. Koo, E. H. Kang, D. H. Kim, K. Bae and J. H. Shim, High-performance protonic ceramic fuel cells with a $\mathrm{PrBa}_{0.5} \mathrm{Sr}_{0.5} \mathrm{Co}_{1.5} \mathrm{Fe}_{0.5} \mathrm{O}_{5+\delta}$ cathode with palladium-rich interface coating, J. Power Sources, 2021, 482, 229043.

4 X. Yao, K. Klyukin, W. Lu, M. Onen, S. Ryu, D. Kim, N. Emond, I. Waluyo, A. Hunt, J. A. del Alamo, J. Li and B. Yildiz, Protonic solid-state electrochemical synapse for physical neural networks, Nat. Commun., 2020, 11, 3134.

5 S. S. Setayandeh, C. J. Webb and E. M. A. Gray, Electron and phonon band structures of palladium and palladium hydride: a review, Prog. Solid State Chem., 2020, 60, 100285.

6 Y. Z. Chen, H. P. Barth, M. Deutges, C. Borchers, F. Liu and R. Kirchheim, Increase in dislocation density in colddeformed $\mathrm{Pd}$ using $\mathrm{H}$ as a temporary alloying addition, Scr. Mater., 2013, 68, 743-746.

7 B. Amin-Ahmadi, D. Connétable, M. Fivel, D. Tanguy, R. Delmelle, S. Turner, L. Malet, S. Godet, T. Pardoen, 
J. Proost, D. Schryvers and H. Idrissi, Dislocation/hydrogen interaction mechanisms in hydrided nanocrystalline palladium films, Acta Mater., 2016, 111, 253-261.

8 T. A. Peters, P. A. Carvalho, J. F. van Wees, J. P. Overbeek, E. Sagvolden, F. P. F. van Berkel, O. M. Løvvik and R. Bredesen, Leakage evolution and atomic-scale changes in Pd-based membranes induced by long-term hydrogen permeation, J. Membr. Sci., 2018, 563, 398-404.

9 R. Griessen, N. Strohfeldt and H. Giessen, Thermodynamics of the hybrid interaction of hydrogen with palladium nanoparticles, Nat. Mater., 2016, 15, 311-317.

10 O. Y. Vekilova, D. I. Bazhanov, S. I. Simak and I. A. Abrikosov, First-principles study of vacancy-hydrogen interaction in Pd, Phys. Rev. B: Condens. Matter Mater. Phys., 2009, 80, 1-5.

11 R. Nazarov, T. Hickel and J. Neugebauer, Ab initio study of $\mathrm{H}$-vacancy interactions in fcc metals: implications for the formation of superabundant vacancies, Phys. Rev. B: Condens. Matter Mater. Phys., 2014, 89, 1-18.

12 J. M. Polfus, O. M. Løvvik, R. Bredesen and T. Peters, Hydrogen Induced Vacancy Clustering and Void Formation Mechanisms at Grain Boundaries in Palladium, Acta Mater., 2020, 195, 708-719.

13 W. Zhong, Y. Cai and D. Tománek, Computer simulation of hydrogen embrittlement in metals, Nature, 1993, 362, 435-437.

14 F. Guazzone and Y. H. Ma, Leak Growth Mechanism in Composite Pd Membranes Prepared by the Electroless Deposition Method, AIChE J., 2008, 54, 487-494.

15 Z. R. Xu and R. B. Mclellan, Hydrogen enhanced diffusional creep, Acta Mater., 1998, 46, 4543-4547.

16 T. B. Flanagan and C. N. Park, Hydrogen-induced rearrangements in Pd-rich alloys, J. Alloys Compd., 1999, 293, 161-168.

17 N. Fukumuro, M. Yokota, S. Yae, H. Matsuda and Y. Fukai, Hydrogen-induced enhancement of atomic diffusion in electrodeposited Pd films, J. Alloys Compd., 2013, 580, S55-S57.

18 A. D. Leclaire and A. B. Lidiard, LIII. Correlation effects in diffusion in crystals, Philos. Mag. A, 1956, 1, 518-527.

19 P. Hänggi, P. Talkner and M. Borkovec, Reaction-rate theory: fifty years after Kramers, Rev. Mod. Phys., 1990, 62, 251-341.

20 M. Mantina, Y. Wang, R. Arroyave, L. Q. Chen, Z. K. Liu and C. Wolverton, First-principles calculation of self-diffusion coefficients, Phys. Rev. Lett., 2008, 100, 1-4.

21 G. Kresse, Efficiency of ab-initio total energy calculations for metals and semiconductors using a plane-wave basis set, Comput. Mater. Sci., 1996, 6, 15-50.

22 J. Perdew, K. Burke and M. Ernzerhof, Generalized Gradient Approximation Made Simple, Phys. Rev. Lett., 1996, 77, 3865-3868.

23 A. Zunger, S.-H. Wei, L. G. Ferreira and J. E. Bernard, Special quasirandom structures, Phys. Rev. Lett., 1990, 65, 353-356.

24 G. Henkelman, B. P. Uberuaga and H. Jónsson, A climbing image nudged elastic band method for finding saddle points and minimum energy paths, J. Chem. Phys., 2000, 113, 9901-9904.

25 C. Zhang and A. Alavi, First-principles study of superabundant vacancy formation in metal hydrides, J. Am. Chem. Soc., 2005, 127, 9808-9817.

26 J.-P. Du, W. T. Geng, K. Arakawa, J. Li and S. Ogata, Hydrogen-Enhanced Vacancy Diffusion in Metals, J. Phys. Chem. Lett., 2020, 11, 7015-7020.

27 G. Boisvert, L. J. Lewis and A. Yelon, Many-Body Nature of the Meyer-Neldel Compensation Law for Diffusion, Phys. Rev. Lett., 1995, 75, 469-472.

28 L. Messina, M. Nastar, N. Sandberg and P. Olsson, Systematic electronic-structure investigation of substitutional impurity diffusion and flux coupling in bcc iron, Phys. Rev. B, 2016, 93, 1-18.

29 A. G. Jones, Compensation of the Meyer-Neldel Compensation Law for $\mathrm{H}$ diffusion in minerals, Geochem., Geophys., Geosyst., 2014, 15, 2616-2631.

30 A. V. Berenov, J. L. MacManus-Driscoll and J. A. Kilner, Observation of the compensation law during oxygen diffusion in perovskite materials, Int. J. Inorg. Mater., 2001, 3, 1109-1111.

31 S. Gelin, A. Champagne-Ruel and N. Mousseau, Enthalpyentropy compensation of atomic diffusion originates from softening of low frequency phonons, Nat. Commun., 2020, 11, 1-7.

32 M. Yoshihara and R. B. McLellan, Self-diffusion in the noble metals, Acta Metall., 1981, 29, 1277-1283.

33 T. R. Mattsson and A. E. Mattsson, Calculating the vacancy formation energy in metals: Pt, Pd, and Mo, Phys. Rev. B: Condens. Matter Mater. Phys., 2002, 66, 1-8.

34 S. L. Shang, B. C. Zhou, W. Y. Wang, A. J. Ross, X. L. Liu, Y. J. Hu, H. Z. Fang, Y. Wang and Z. K. Liu, A comprehensive first-principles study of pure elements: vacancy formation and migration energies and self-diffusion coefficients, Acta Mater., 2016, 109, 128-141.

35 W. Huang, S. M. Opalka, D. Wang and T. B. Flanagan, Thermodynamic modelling of the Cu-Pd-H system, Comput. Coupling Phase Diagrams Thermochem., 2007, 31, 315-329.

36 N. L. Peterson, Isotope Effect in Self-Diffusion in Palladium, Phys. Rev., 1964, 136, 568-574.

37 J. Fillon and D. Calais, Autodiffusion dans les alliages concentres fer-palladium, J. Phys. Chem. Solids, 1977, 38, 81-89.

38 D. K. Hsu and R. G. Leisure, Elastic constants of palladium and $\beta$-phase palladium hydride between 4 and 300 K, Phys. Rev. B: Condens. Matter Mater. Phys., 1979, 20, 1339-1344.

39 W. Zhong, Y. Cai and D. Tománek, Mechanical stability of Pd-H systems: a molecular dynamics study, Phys. Rev. B: Condens. Matter Mater. Phys., 1992, 46, 8099-8108.

40 N. I. Timofeev, F. N. Berseneva and V. M. Makarov, New palladium-based membrane alloys for separation of gas mixtures to generate ultrapure hydrogen, Int. J. Hydrogen Energy, 1994, 19, 895-898. 
41 J. Okazaki, D. A. P. Tanaka, M. A. L. Tanco, Y. Wakui, F. Mizukami and T. M. Suzuki, Hydrogen permeability study of the thin Pd-Ag alloy membranes in the temperature range across the $\alpha-\beta$ phase transition, J. Membr. Sci., 2006, 282, 370-374.
42 I. Coulthard and T. K. Sham, Charge redistribution in Pd-Ag alloys from a local perspective, Phys. Rev. Lett., 1996, 77, 4824-4827.

43 R. B. McLellan and D. Zang, Kinetics of vacancy migration in hydrogenated palladium, Scr. Mater., 1997, 36, 1207-1210. 